\title{
Laser Therapy for Vitiligo
}

Angelo Massimiliano D’Erme ${ }^{1 *}$, Valeria Mateeva ${ }^{2}$, Zoulikha Zarrab ${ }^{3}$ and Torello Lotti ${ }^{4}$

${ }^{1}$ Division of Dermatology, University of Florence, Italy

${ }^{2}$ Clinic of Dermatovenerology and Allergology, Military Medical Academy Sofia, Bulgaria

${ }^{3}$ Saint-Petersburg State Pediatric Medical University, Saint Petersburg, Russia

${ }^{4}$ Chair of Dermatology, University of Rome "G. Marconi", Rome, Italy

\section{Introduction}

Vitiligo is a common, acquired pigmentary disorder, characterized by variable-sized depigmented patches, of unknown etiology affecting $1-2 \%$ of world population without any racial, geographic or sexual predilection. The course of disease is chronic, and often progressive [1].

The current treatment options for vitiligo are not really satisfactory to the patient population and the dermatologists [2].

The current paper aims to give an overview of the effectiveness of laser treatments alone or in combination with topical treatment in the management of vitiligo lesions.

At the moment the laser therapy consists of UVB at $308 \mathrm{~nm}$ and a UVB $311 \mathrm{~nm}$ narrow-band microphototherapy that are indicated for segmental and non-segmental vitiligo. UVA treatments represent a second line treatment in vitiligo even thought new devices have been introduced in the market. Lastly authors report the results of recent studies for the use of other lasers in vitiligo therapy.

\section{UVB Laser Therapy and Phototherapy}

UVB laser therapy and narrow band (nb)-UVB phototherapy are suggested for vitiligo lesions that involve more than $15-20 \%$ of the body area. UVB-laser therapy represents a therapeutic option also for lesions of smaller involvement that are actively spreading on the body.

UVB-laser therapy at the wavelength of 308 and $311 \mathrm{~nm}$ represents a targeted therapy in the treatment of vitiligo. It represents a therapeutic option for the treatment of vitiligo resistant to treatment, in alternation or in combination with topical treatment.

The choice of narrow-band UVB-laser therapy or localized or whole-body narrow-band UVB phototherapy depends on the extension and involvement of vitiligo and the devices available.

As also reported in recent guidelines [2] they represent the first choice for localized vitiligo in particular for small lesions of recent onset and childhood vitiligo.

The combination with topical steroid or topical calcineurin inhibitors improves the clinical outcome $[3,4]$.

As reported by Lotti et al., $0.05 \%$ betamethasone dipropionate cream plus $311 \mathrm{~nm}$ narrow-band UVB microfocused phototherapy give higher repigmentation rate then monotherapy with $311 \mathrm{~nm}$ narrowband UVB [5].

The use of targeted laser therapy has the advantage to avoid the patient aging and the risk of skin cancer associated with total body irradiation.

Furthermore, also the side effects of the treatment, as erythema and burning, are localized to a confined area.

\section{UVA Laser Therapy and Phototherapy}

In the last decade, light therapy lamps with halogen-metal band confined to the very high irradiance UVA1 (340-400 $\mathrm{nm}$ ) has experienced a growing interest and use in dermatology.
The immunomodulating effects of UVA1 are mainly mediated by aerobic photo-oxidation. The intermediate formation of reactive oxygen species induces the apoptosis of T lymphocytes [6].

The treatment with high-intensity UVA phototherapy plus Psoralene (PUVA), acts though stimulation of inactive melanocytes in the outer root sheath of hair follicles to proliferate, mature, and migrate to repopulate the interfollicular epidermis.

UVA phototherapy (320-400 nm) can be combined with oral (PUVA) or topical (TUVA) administration of psoralens photosensitizers. Topical psoralen lotions can be administered to patients suffering from vitiligo affecting less than $10 \%$ of the body. This lotion is applied on the interested area of the skin 15 to 30 minutes before the exposure to UVA rays.

It can be used as an alternative to PUVA in patients with hepatic dysfunction or gastric disturbances $[3,6]$.

A more interesting combination is represented by the treatment with UVA phototherapy in association with medium potency topical corticosteroid. This combination treatment has been reported to be about three times more effective than either treatment alone, suggesting synergy between melanocyte stimulation and immunosuppressive effects.

Nowadays, oral PUVA is currently used in adult patients with generalized vitiligo as a second-line therapy. In fact, compared with nbUVB it has the disadvantage of lower efficacy and higher short- and long-term risks [2]. As with NB-UVB,

12-24 months of continuous therapy may be necessary to acquire maximal repigmentation.

Recently, a new instrument, laser Alba $355^{\circ}$, has been commercialized. This instrument is based on a 1064 wavelength neodymium-doped yttrium orthovanadate (Nd:YVO4) laser optically pumped using a $808 \mathrm{~nm}$ infrared beam able to achieve a third harmonic $355 \mathrm{~nm}$ wave delivery. It administers energy in the UVA1 emitting wavelengths mainly around 355nm [7,8].

Two studies have recently demonstrated the efficacy of $355 \mathrm{~nm}$ UVA1 laser therapy in psoriasis, but also other immune-mediated skin disorders, as atopic eczema, hand eczema, localized scleroderma, mycosis fungoides and vitiligo may benefit from its use [6-8].

Localized UVA-1 laser therapy has the advance to treat only the involved area: preliminary experience support its use also in vitiligo.

*Corresponding author: Angelo Massimiliano D’Erme, Division of Dermatology, University of Florence, Viale Michelangiolo 41, 50129 Florence, Italy, Tel: +393498451303; Fax: +390556939595; E-mail: a.m.derme@gmail.com

Received: October 20, 2014; Accepted: October 25, 2014; Published: October 27, 2014

Citation: D'Erme AM, Mateeva V, Zarrab Z, Lotti T (2014) Laser Therapy for Vitiligo. Pigmentary Disorders 1:144. doi: 10.4172/JPD.1000144

Copyright: () 2014 D'Erme AM, et al. The terms of the Creative Commons Attribution License, which permits unrestricted use, distribution, and reproduction in any medium, provided the original author and source are credited. 


\section{Other Laser Treatments}

Fractional $\mathrm{CO}_{2}$ laser is a new method that can be proposed in the management of vitiligo in selected patients who do not have received any evident benefit from previous treatments [2].

Either alone or in combination with other treatments, fractional $\mathrm{CO}_{2}$ laser treatment has been reported to induce benefits in repigmentation of vitiliginous lesions.

The association of NB-UVB with fractional $\mathrm{CO}_{2}$ laser has been reported to be effective in refractory areas as reported in a small randomized left-right comparative trial [9].

In widespread vitiligo, where repigmentation terapie are ineffective, depigmentation can also be obtained by using a Q-switched ruby laser, alone or in combination with methoxyphenol [2].

\section{References}

1. Taïeb A, Picardo M (2009) Clinical practice. Vitiligo.N Engl J Med 360: 160-169.

2. Taieb A, Alomar A, Böhm M, Dell'anna ML, De Pase A, et al. (2013) Guidelines for the management of vitiligo: the European Dermatology Forum consensus. Br J Dermatol 168: 5-19.
3. Lotti T, Tripo L, Grazzini M, Krysenka A, Buggiani G, et al. (2009) Focused UV-B narrowband microphototherapy (Biopsorin). A new treatment for plaque psoriasis.Dermatol Ther 22: 383-385.

4. Hercogová J, Buggiani G, Prignano F, Lotti T (2007) A rational approach to the treatment of vitiligo and other hypomelanoses.Dermatol Clin 25: 383-392, ix.

5. Lotti T, Buggiani G, Troiano M, Assad GB, Delescluse J, et al. (2008) Targeted and combination treatments for vitiligo. Comparative evaluation of different current modalities in 458 subjects.Dermatol Ther 21 Suppl 1: S20-26.

6. Gambichler T, Terras S, Kreuter A (2013) Treatment regimens, protocols dosage, and indications for UVA1 phototherapy: facts and controversies.Clin Dermatol 31: 438-454.

7. Nisticò SP, Saraceno R, Chiricozzi A, Giunta A, Di Stefani A, et al. (2013) UVA-1 laser in the treatment of palmoplantar pustular psoriasis.Photomed Laser Surg 31: 434-438.

8. Zerbinati N, Portale S, Palmieri B, Rottigni V, lannitti T (2012) A preliminary study to assess the efficacy of a new UVA1 laser for treatment of psoriasis. Photomed Laser Surg 30: 610-614

9. Tsukamoto K, Osada A, Kitamura R, Ohkouchi M, Shimada S, et al. (2002) Approaches to repigmentation of vitiligo skin: new treatment with ultrasonic abrasion, seed-grafting and psoralen plus ultraviolet A therapy.Pigment Cell Res 15: 331-334. 